\title{
Enseñanza de habilidades argumentativas básicas a través de un juego serio on line.
}

\section{Teaching Basic Argumentative Skills Through a Serious Online Game}

Jorge Iván Hoyos-Morales

Secretaria de Educación Municipal

Cartago, Colombia

Investigador Externo CETIE - UVa

jorgeivanhoyos@gmail.com

\section{RESUMEN}

Este trabajo se inscribe en la línea investigativa enseñar a argumentar, enfocándose en las habilidades argumentativas y vinculando los juegos serios on line. Los objetivos fueron: primero, explorar un juego serio online para la enseñanza de habilidades argumentativas básicas. Segundo, observar la progresión de los estudiantes en el domino de tales habilidades al interactuar con el juego. Se adoptó como método el estudio de caso exploratorio mixto, con alumnos del penúltimo grado de educación media en un colegio estatal colombiano. Uno de los resultados más relevantes fue que el $61,36 \%$ de los estudiantes mejoraron su nivel de dominio en al menos una de las habilidades observadas.

PALABRAS CLAVE: enseñanza de la argumentación, habilidades argumentativas, juegos serios, progresiones de aprendizaje, quandary.

\section{ABSTRACT}

This work is related to the research line teaching argumentation, focusing on argumentative skills and linking serious online games. The objectives were: first, to explore a serious online game for teaching the basic argumentative skills. Second, observe the students' progression in the mastery of such skills when they interact with the game. The mixed exploratory case study was adopted as a method, with students from the penultimate grade of secondary education in a Colombian state school. One of the most relevant results was that $61,36 \%$ of the students improved their level of proficiency in at least one of the skills observed.

KEYWORDS: argumentative skills, learning progressions, Quandary, serious on line games, teaching of argumentation. 


\section{INTRODUCCIÓN}

\subsection{Procesos educativos y argumentación}

Ciertamente «la preocupación por cómo argumentamos y el papel de la educación en el desarrollo de las habilidades argumentativas tiene (...) una larga historia» (PérezEcheverría, Postigo, \& Garcia-Mila, 2016, pág. 11). En tal preocupación están presentes los procesos de enseñanza-aprendizaje que reputados autores «consideran el centro de la investigación y la práctica didáctica» (Gimeno Sacristán \& Pérez Gómez, 1992, pág. 34).

En ese sentido investigadores como von Aufschnaiter, Erduran, Osborne, y Simon (2008), Schwarz (2009) y Schwarz \& Asterhan (2016) identifican dos líneas investigativas: aprender a argumentar y argumentar para aprender; las cuales se centran en el rol del estudiante. La primera línea «implica la adquisición de herramientas generales como justificar, desafiar, responder el desafío o conceder» (Schwarz, 2009, pág. 92) La segunda línea «a menudo se ajusta a un objetivo específico cumplido a través de la argumentación, y en un marco educativo, la meta (implícita) es entender o construir conocimientos específicos» (Schwarz \& Asterhan, 2016, pág. 92).

Sin embargo no se puede olvidar que para fines investigativos es posible distinguir entre proceso de aprendizaje y proceso de enseñanza, y por ende resulta plausible diferenciar otras dos líneas investigativas: enseñar a argumentar y enseñar argumentando, en las que aparece como sujeto agente el educador.

En tales líneas el educador al enseñar tiene determinadas intenciones y "en ese orden de ideas enseñar a argumentar hace referencia a aquella intención docente que en determinado caso se interesa por ofrecer a los alumnos elementos para que puedan construir, analizar o evaluar discursos argumentativos." (Hoyos-Morales, 2018, pág. 98). Cabe anotar que enseñar a argumentar parece necesario "para que la argumentación pueda usarse como herramienta de aprendizaje" (Pérez-Echeverría, Postigo, \& GarciaMila, 2016, pág. 16). Por su parte el enseñar argumentando hace referencia:

[e]n primer lugar al acompañamiento realizado por el profesor para que el estudiante comunique de manera razonada el saber disciplinar adquirido. En segundo lugar enseñar argumentando se refiere a los diversos recursos argumentativos de los cuales el docente echa mano para trasmitir un contenido o para persuadir, esto es para formar un juicio en sus estudiantes. (HoyosMorales, 2018, pág. 99).

De lo anterior se desprende que es posible hacer investigaciones que relacionen 
procesos de enseñanza-aprendizaje con la argumentación siguiendo cuatro líneas; una de las cuales será la que se explore en el presente artículo: la enseñanza de la argumentación o mejor la enseñanza de habilidades argumentativas.

\subsection{Enseñanza de habilidades argumentativas}

Para abordar con amplitud el tema anunciado es necesario tener en cuenta tanto la mirada que se tiene desde el ámbito educativo, como la que se plantea desde la teoría de la argumentación.

Desde el punto de vista educativo las habilidades argumentativas han despertado interés en los investigadores psicoeducativos por su importancia «para la reconceptualización del aprendizaje en las disciplinas escolares» (Schwarz \& Asterhan, 2016, pág. 164). Muestra de ello es que tales habilidades resultan fundamentales para constituir procesos psicológicos superiores o para alcanzar niveles cognitivos complejos como evaluar en una asignatura (Bloom, 1956, pág. 207). Cabe anotar que los procesos y niveles antedichos implican tanto la capacidad de razonar (Baquero, 1997, pág. 45), como la precisión lógica, el reconocer hechos, documentar pruebas y la «habilidad para indicar las falacias lógicas en los argumentos» (Lorin \& Krathwohl , 2001, pág. 277).

En segundo lugar, enseñar habilidades argumentativas es útil para desarrollar metacognición ya que esta implica el conocimiento de estrategias genéricas para pensar inductiva o deductivamente, tales como «evaluar la validez de diferentes enunciados lógicos, evitar la circularidad en los argumentos, hacer inferencias apropiadas de diferentes fuentes de datos y recurrir a muestras apropiadas para hacer inferencias» (Pintrich, 2002, pág. 221).

En tercer lugar autores como Matthew Lipman, Richard Paul y Linda Elder consideran que las habilidades argumentativas son parte fundamental del enseñar a pensar críticamente. Por ejemplo, Lipman et al. (1992) colocan la mejora de la capacidad de razonar entre los fines y objetivos de su Filosofía para Niños pues consideran que «los niños pueden tener problemas con las inferencias perceptivas, las inferencias lógicas o las inferencias empíricas» (p. 141), lo cual probablemente les acarree dificultades académicas. Por su parte Paul y Elder, conscientes de la importancia del pensamiento crítico para estar presentes «en un mundo repleto de miedo e inseguridad» (2005, pág. 12), se interesan en las habilidades para pensar críticamente distinguiendo el pensamiento crítico débil y el fuerte. El primero consiste en la habilidad «de formular razones válidas, de combinarlas con argumentos bien estructurados, refutar contra 
argumentos, etcétera» (Perkins, 2001, pág. 117). El segundo tiene que ver con disposiciones como el compromiso y la voluntad por mantener el pensamiento abierto a perspectivas distintas, cultivar la curiosidad intelectual, buscar la claridad, etc... Podría decirse que el pensamiento crítico fuerte se relaciona estrechamente con el cultivo de virtudes argumentativas (Aberdein, 2010) que incluyen «aquellas disposiciones estables referidas a la voluntad de participar en la argumentación, escuchar, modificar el propio punto de vista o cuestionar lo obvio» (Hoyos-Morales, 2018, pág. 300).

En cuarto lugar, enseñar habilidades argumentativas ayuda a hacer visible el pensamiento, que es un objetivo propio de la enseñanza para la comprensión. Este enfoque incluye rutinas de pensamiento entendidas como «patrones sencillos de pensamiento que pueden ser utilizados una y otra vez» (Perkins, 1997, pág. 4). En ese sentido Perkins encuentra muy útil una rutina que «incluye dos preguntas clave: ¿Qué está sucediendo en esta situación? y ¿Qué observas que te lleva a decir eso?» (ibíd.) Tal rutina supone enseñar habilidades argumentativas pues «a medida que los estudiantes responden, el docente fácilmente puede clasificar las sugerencias como hipótesis y sus comentarios de apoyo como razones» (ibíd.) hasta que tal patrón se convierta en parte del pensamiento del estudiante.

Adicionalmente es interesante señalar que, desde la perspectiva de las inteligencias múltiples, la enseñanza de las habilidades en mención resulta ser clave «para una programación didáctica que comprenda tanto metas claras, como medios disponibles y formas de evaluación adecuadas» (Hoyos-Morales, 2018, pág. 168) relativas al desarrollo elementos propios de la inteligencia lingüística, tales como la habilidad «para convencer a otros individuos acerca de un curso de acción» (Gardner, 2001, pág. 71) o la habilidad para explicar un determinado asunto.

Ahora bien, cuando se plantea como meta educativa que los estudiantes comprendan, evalúen y critiquen lo que leen; la tarea de «describir objetivos conductuales que cubran todas y cada una de las metas puede llegar a convertirse en una tarea agobiante, por no decir imposible» (Ormrod, 2005, pág. 97). Frente a esta situación, enumerar habilidades argumentativas proporciona una idea más precisa de lo que se quiere alcanzar. Algunas de tales habilidades, según Ormrod podrían ser: distinguir entre hechos, opiniones, inferencias, supuestos y conclusiones; identificar relaciones causa-efecto o errores en el razonamiento.

No se puede dejar de mencionar que la enseñanza de las habilidades argumentativas es importante para los psicopedagogos, porque ante «la vasta cantidad 
de información causada por la creciente accesibilidad a las modernas tecnologías de la comunicación... [puede ayudar a lograr] una exitosa participación en las sociedades democráticas del siglo XXI» (Schwarz \& Asterhan, 2016, pág. 164).

Desde el punto de vista de los estudiosos de la argumentación, autoras como como Muller Mirza y Perret-Clermont plantean que la:

[a]rgumentación tiene una importancia creciente en la educación, no solo porque es una competencia importante que debe aprenderse, sino también porque la argumentación puede utilizarse para fomentar el aprendizaje en filosofía, historia, ciencias y matemáticas, y en muchos otros dominios (Muller Mirza \& PerretClermont, 2009, pág. 1).

En tal sentido Siegel manifiesta que la enseñanza de la argumentación, y podría decirse que la enseñanza de habilidades argumentativas, es importante para los educadores dado que «el objetivo de la educación consiste en desarrollar la racionalidad de los estudiantes» (1995, pág. 159).

Por su parte D. Kuhn pone como premisa que «los estudiantes no solo necesitan argumentar sino también aprender a argumentar bien» (2008, pág. 113) sin embargo los profesores no conocen muy bien la manera de hacerlo. En ese sentido, fruto de sus investigaciones, la investigadora en mención (2008, pág. 153) plantea un conjunto de actividades que pueden constituir una progresión para la enseñanza de habilidades argumentativas: generar y elaborar buenas razones que apoyen las opiniones; apoyar razones con evidencias; evaluar cuales razones son mejores que otras; conectar razones para construir bloques argumentativos; examinar y evaluar las razones opuestas; generar contra argumentaciones; usar la evidencia para apoyar diferentes puntos de vista; evaluar qué argumentos son más fuertes que otros.

También C. Plantin (2014), basándose en la argumentación desde el lenguaje, y partiendo de su experiencia tanto docente como investigativa, asevera que «un porcentaje importante de estudiantes... distingue mal, en un texto, lo que es la conclusión, la tesis defendida, la posición del locutor, y, por otra parte, los argumentos, las buenas razones que presenta a favor de esa tesis» (Warley, 2011, pág. 111); tal situación interpela a quienes laboran en el ámbito educativo y hace pensar en la importancia de enseñar a argumentar.

Para terminar este apartado de introducción temática, cabe anotar que lo planteado por Kuhn y lo aseverado por Plantin se inserta en el dominio práctico que el enfoque pragmadialéctico plantea para su programa de investigación en argumentación. En este dominio se tiene como objetivo «incrementar metódicamente las destrezas y 
habilidades de las personas tanto para producir discursos argumentativos como para analizarlos y evaluarlos» (van Eemeren \& Grootendorst, 2004, pág. 28); para lograr dicho objetivo se necesitan proponer programas de mejoramiento de modo que las personas argumenten de manera razonable, o estrategias que incrementen 0 consoliden sus habilidades argumentativas, iniciando por las básicas tales como la diferenciación de hechos, soluciones, opiniones; o la identificación de argumentos a favor y argumentos en contra.

\subsection{TICs y juegos serios en la educación}

El otro gran aspecto del tema abordado en este trabajo son las nuevas tecnologías de la información y la comunicación, las cuales se han ido incorporando al ámbito educativo. Sin embargo hay que subrayar que tal incorporación debe ser fruto de una decisión razonada y razonable; pues de lo contrario se inicia con «unas altas expectativas que al no cumplir lo prometido hacen que se retorne, con aire de decepción o derrota, a las prácticas tradicionales» (Hoyos-Morales, 2018, pág. 68).

Para hacer frente a lo anterior resulta interesante «la propuesta construccionista de Seymour Papert, quien parte de postulados piagetianos, de la teoría computacional y la inteligencia artificial» (Hoyos-Morales, 2018, pág. 69), para desarrollar el lenguaje de programación Logo que está a la base tanto del software Micromundos, como de otro lenguaje llamado Scracth «que casi todos los niños [encuentran] compatible con sus fines, fortalezas e imaginación. [Adicionalmente,] los fines educativos y prioridades no son dictaminados desde arriba; más bien emergen a partir de la exploración del entorno Scratch que hace el niño» (Gardner \& Davis, 2013, pág. 148). Tales herramientas se desarrollaron en el Laboratorio de Aprendizaje Construccionista, entre cuyos principios de trabajos están: la diversión difícil, la conciencia que estamos entrando en el mundo digital, y el aprender aprender (Papert, 2013, pág. 71). Así las cosas, el construccionismo de Papert muestra una manera de usar razonada y razonablemente las TICs en educación.

Es de resaltar que el principio de diversión difícil y el de la entrada en el mundo digital dan cuenta de la inclinación por introducir la gamificación o ludificación y los juegos serios en educación; lo cual no es un capricho sino un responder a una tendencia social empíricamente constatable, pues «de hecho los gamers... gastan más de diez 10.000 horas jugando, hasta la edad de 21 años; lo que equivale al tiempo invertido en educación desde la primaria hasta la secundaria» (Ciftci, 2018, pág. 18). 
Lo anterior muestra la importancia que tienen los juegos digitales para los jóvenes en edad escolar, sin embargo es importante aclarar que gamificación y uso de juegos serios no son sinónimos. Mientras lo primero se refiere «a la conversión en juego de algo que inicialmente no fue pensado como tal, con la finalidad de llevar a cabo algún aprendizaje» (Rodriguez Gomez \& Ibarra Sáiz, 2014, pág. 12); lo segundo hace referencia a pruebas mentales o simulaciones creadas con objetivos y reglas específicas que usan el juego para aprender contenidos disciplinares o habilidades. En ese sentido, hay que precisar que «El juego es educativo sólo cuando "esconde" algunos objetivos de aprendizaje. Es importante, sin embargo [...] que el juego siga siendo divertido» (Rugelj, 2014, pág. 33) y conserve sus características en cuanto juego; es decir que cuente una historia, que tenga reglas estructurantes, que esté provisto de un entorno donde el jugador interactúe, que dé satisfacción y que brinde retroalimentación tanto al jugador como al profesor.

Con base en todo lo dicho hasta aquí, este artículo persigue un doble objetivo: por una parte explorar un juego serio online para la enseñanza de habilidades argumentativas básicas. Por otra parte observar la progresión de los estudiantes en el domino de habilidades argumentativas básicas al interactuar con dicha herramienta. Las habilidades a observar son por un lado la identificación de hechos, opiniones y soluciones; y por el otro la clasificación de argumentos a favor y en contra.

\section{MÉTODO}

Para dar cuenta de los objetivos planteados se elige el estudio de caso exploratorio (Yin, 2003) con tratamiento mixto de los resultados en dos fases secuenciales: una cualitativa y otra cuantitativa (Creswell, 2009, pág. 209). Tal método es útil para mostrar intervenciones en contextos reales comprendiéndolas desde lo cualitativo y analizándolas desde lo cuantitativo. Cabe anotar que el contexto en el que se realiza el presente trabajo es el de las clases de filosofía, realizadas durante los meses de agosto, septiembre y octubre del año 2018 con 102 adolescentes que cursaban el penúltimo año de educación media en el colegio estatal Colombiano Antonio Holguín Garcés, situado en la localidad de Cartago, Valle. Debe añadirse que desde el punto de vista didáctico se tiene como premisa que la filosofía puede contribuir al desarrollo del pensamiento crítico, el cual supone que las personas poseen cierto domino de habilidades argumentativas básicas.

Para este estudio de caso se recurrieron a dos fuentes de información principales: 
por una parte a trabajos previos relativos a la enseñanza de la argumentación y por otra a la observación indirecta de la interacción de los estudiantes con el juego serio on line elegido. Adicionalmente, como se suele hacer en los estudios de caso, se llevó un diario de campo del investigador y se aplicó una encuesta a estudiantes que interactuaron con el juego. Es importante señalar que se hizo un análisis estadístico descriptivo de lo observado con el fin de obtener resultados y discutir tanto los antecedentes como los datos.

\subsection{Trabajos previos}

Se considera oportuno acudir a la investigación acerca de las implicaciones para la enseñanza que tiene la argumentación en clase (Hoyos-Morales, 2018), pues en dicho trabajo se presenta un estado del arte acerca de las relaciones entre la argumentación y los procesos de enseñanza y aprendizaje. Para construir tal estado del arte se acudió a bases de datos especializadas como EBSCO, ERIC, GOOGLE SCHOLAR, SCOPUS y WOS; las palabras clave usadas fueron: aprender a argumentar, aprender argumentando, enseñar a argumentar y enseñar argumentando; tanto en inglés como en español. Posteriormente se filtraron los trabajos para tener en cuenta aquellos realizados a partir de 2007, enfocados en el nivel secundario de enseñanza y relacionados o relacionables con las asignaturas de Historia, Lenguaje y Sociales. Respecto a enseñar a argumentar allí se encontraron: una revisión histórica, dos artículos originales de corte teórico y diez artículos de corte teórico-práctico clasificables o como estudios de caso o centrados en metodologías. A continuación se mencionarán los trabajos que más ayudaron a la construcción del presente estudio de caso.

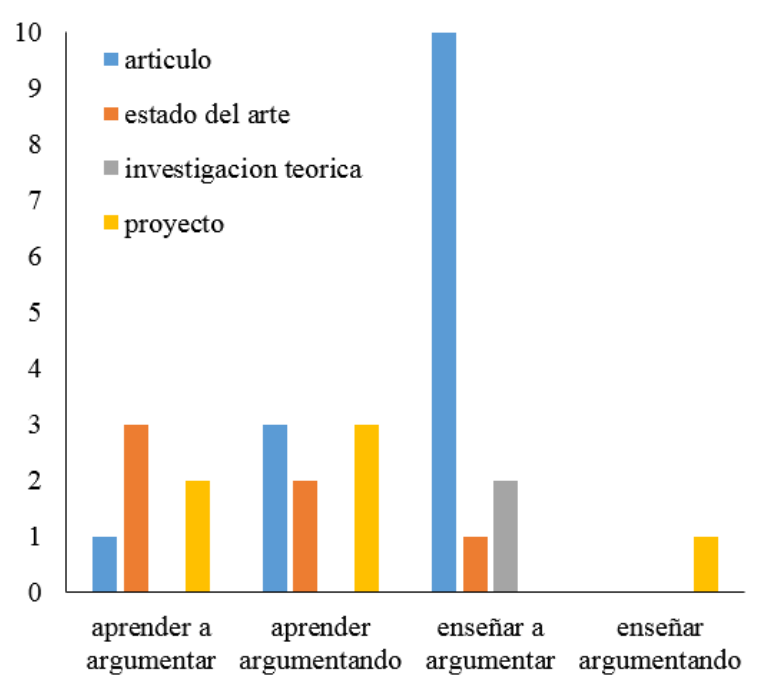

Figura 1 Relación argumentación-educación Fuente: (Hoyos-Morales, 2018, pág. 100) 
El estado del arte de Guzmán-Cedillo, et al. (2013) «plantea la necesidad de revisión, definición y caracterización de la competencia argumentativa... a partir de la revisión de documentos... [Y llega a plantear] la competencia argumentativa como la articulación de... conocimientos, habilidades y actitudes» (pág. 907) que pueden ser educables. Cabe decir que en su comprensión de competencia argumentativa, los autores recurren a enfoques como los de van Eemeren, Toulmin y Perelman; pero también a los trabajos empíricos de D. Kuhn.

Desde el punto de vista teórico Deane \& Song (2014) Por una parte presentan «un modelo para evaluar la progresión de habilidades para la lectura y escritura argumentativa. [Por otra parte] identifican que para involucrar a los estudiantes en la construcción de argumentaciones es importante realizar diferentes actividades» (HoyosMorales, 2018, pág. 112) analizables en un ciclo argumentativo estructurado a partir de las tareas que la retórica clásica asigna al sujeto que argumenta; es decir inventio, dispositio y elocutio. Las etapas de tal ciclo son: «1) comprender lo demandado por la audiencia; 2) explorar el tema; 3) considerar los distintos puntos de vista; 4) crear y evaluar argumentos; 5) organizar y presentar la argumentación» (ibíd.). En cada etapa se manifiestan habilidades sociales, conceptuales y discursivas que cambian dependiendo del nivel de escolaridad y del proceso cognitivo involucrado (interpretación, expresión o deliberación). Estos investigadores manifiestan que su modelo «puede contribuir a que los profesores tomen decisiones instruccionales que apoyen de modo efectivo el desarrollo de habilidades... de sus estudiantes» (Hoyos-Morales, 2018, pág. 88).

Rijn et el (2014) validan el modelo teórico antedicho, determinando las progresiones en habilidades argumentativas de 1840 estudiantes de enseñanza secundaria obligatoria. Los investigadores concluyen que el modelo "sirve para clasificar de forma consistente a los estudiantes en los niveles de la progresión de aprendizaje de la capacidad para argumentar" (2014, pág. 115); lo cual es útil para que un profesor pueda «confirmar el nivel o punto de partida de los estudiantes y... su posterior seguimiento formativo» (ibíd.)

Centrándose más en la práctica del enseñar a argumentar, Glassner (2017) plantea que los principales criterios para determinar que es un buen argumento son «claridad, explicitud, relevancia de las razones que apoyan la pretensión, inclusión de evidencia, explicación teórica, aceptabilidad (...) y suficiencia» (pág. 35). En la medida en que tales criterios se aplican, la persona adquiere habilidad por ejemplo para diferenciar hechos de opiniones o para valorar argumentos a favor y en contra. 
Ehrenworth (2017) «defiende la tesis según la cual debido al constante bombardeo de información nunca había sido tan importante enseñar a los jóvenes» (Hoyos-Morales, 2018, pág. 114) ciertas habilidades argumentativas. Tal preocupación también late en el trabajo Jackson, B. \& Wallin, J. (2009), pues «los autores observando las dinámicas comunicativas presentes en plataformas como YouTube se plantean la pregunta acerca de cómo enseñar a los estudiantes a deliberar públicamente» (HoyosMorales, 2018, pág. 118).

En consonancia con lo anterior Kuhn et al (2016), reconociendo la importancia de la argumentación en el ámbito educativo, señalan «que para los profesores enseñar la habilidad de escribir argumentativamente es una de las más desafiantes y en la que los estudiantes muestran bajos desempeños» (Hoyos-Morales, 2018, pág. 116). Por ello estos «investigadores exponen un método para fomentar en adolescentes la habilidad argumentativa» (ibíd.); dicho método involucra el uso de un chat «para promover y facilitar la reflexión» (Kuhn, Hemberger, \& Khait, 2016, pág. 40). Cabe decir que tal método arroja resultados satisfactorios respecto a la comprensión y evaluación de las argumentaciones.

Por su parte Brown (2016) piensa que el aprendizaje dialógico fomenta prácticas argumentativas como la contra-argumentación, rechazo y evaluación de puntos de vista (pág. 82). Leitão (2012) reporta una experiencia innovadora realizada en la universidad en la que utiliza el modelo de debate crítico (Fuentes Bravo \& Santibañez Yañez, 2010) como práctica pedagógica. En Van der Heide et al. (2016) se critica el enfoque formalista de enseñar a argumentar por escrito y en el que se sigue un esquema predeterminado; dado que tal esquema «no persuade a los estudiantes acerca de la significación social y ética de la argumentación» (Hoyos-Morales, 2018, pág. 117). Finalmente el trabajo de Øgreid \& Hertzberg (2009) señala «la importancia del apoyo entre los profesores para la enseñanza de la argumentación escrita» (Hoyos-Morales, 2018, pág. 118) y fruto de ello concluye que «la enseñanza de la argumentación debería incluir explicaciones explicitas de los procesos a aprender; planificar qué tan extensa, elaborada y jerárquica debe ser; la identificación de los objetivos; y el andamiaje de estructuras y dispositivos que ayuden a la escritura argumentativa» (Øgreid \& Hertzberg, 2009, pág. 467).

\subsection{Juego serio elegido}

De acuerdo con el tema y los objetivos de este trabajo se encontró una herramienta que reúne los requisitos para ser calificada como un juego serio que busca desarrollar habilidades argumentativas básicas y que además tiene sintonía con algunos de los 
trabajos citados; se trata de Quandary, juego que ha sido galardonado con varios premios a nivel internacional desde el año 2013.

En primer lugar, hay que decir que Quandary fue diseñado como un juego digital basado en cartas con las cuales el jugador afronta cuatro episodios o escenarios en donde hay dilemas o mejor retos éticos, cognitivos y procedimentales; con tales cartas se deben superar cuatro retos por episodio. En cuanto juego serio, tiene un claro propósito educativo: «apoyar el desarrollo de la toma de decisiones éticas, de pensamiento crítico y de habilidades para la toma de perspectiva» (Learning Games Network, 2019, pág. 2), tanto en niños como en jóvenes. Cabe anotar que el juego es libre y puede catalogarse como aplicación cliente-servidor, pues los usuarios para ingresar tienen un nombre y una contraseña asignada por su profesor, quien previamente se ha registrado como tal. Adicionalmente, en la versión usada, la interface está en tanto en inglés como en español.

En segundo lugar, Quandary cuenta la historia de la primera colonia terrícola instalada a 32 millones de años luz de la Tierra, en el plantea Braxos. Allí los colonos, con la ayuda de su capitán, enfrentan situaciones problemáticas que necesitan resolver: 1) preservar sus animales domésticos de los depredadores locales, a los cuales se les puede investigar para encontrar medicinas. 2) El agua del pozo común parece estar contaminada y por ello se debe comprar agua sacada de un pozo particular; pero no todos los colonos pueden pagar por el agua limpia. 3) El sastre de la colonia hace cambios de diseño en los uniformes de sus amigos, lo que divide a la comunidad. 4) En el tablero comunitario digital aparecen mensajes que molestan a algunos y los colonos deben discernir si se trata de un acoso o de una broma.

En tercer lugar, Quandary tiene unas sencillas reglas estructurantes: 1) el alumno entra con un nombre de usuario y una contraseña que asigna el docente; 2) el alumno toma el rol de líder de la colonia; 3) en cuanto líder, el estudiante debe tomar decisiones difíciles por el bien de la colonia.

En cuarto lugar, el alumno encuentra en cada episodio una situación problema e interactúa con el entorno digital jugando sus cartas, para afrontar cuatro retos: 1) identificar cuáles de las cartas se refieren a hechos, cuales a opiniones y cuales a soluciones; eligiendo posteriormente las dos soluciones que considera más pertinentes. 2) Entender los puntos de vista de cada colono, comparando alternativamente las cartas que refieren a las opiniones de los colonos con las cartas de soluciones elegidas y con hechos que podrían alterar sus opiniones. 3) Habiendo hecho la indagación anterior, el 
jugador se decanta por una solución al problema y apoya tal decisión con cartas que usa como argumentos a favor o en contra de tal solución. Con todos estos datos, el alumno recibe retroalimentación del programa, bajo la forma de un dictamen dado por el consejo colonial que está en la Tierra al tanto de la situación. 4) Finalmente, el capitánjugador confronta la decisión del consejo colonial con las opiniones de los colonos, clasificando las cartas de opinión en aquellas que están en desacuerdo y aquellas que están de acuerdo con el pronunciamiento del consejo colonial.

En quinto lugar, este juego no olvida, en cada episodio, brindar satisfacción a los jugadores en los retos que va afrontando (identificar hechos, opiniones y soluciones; investigar puntos de vista; diferenciar argumentos a favor y en contra; y clasificar acuerdos y desacuerdos). En ese sentido el alumno va obteniendo puntajes de acuerdo con la congruencia de las elecciones que va realizando. Es interesante decir que los episodios concluyen mostrando el resultado final y el impacto en la colonia; adicionalmente cada episodio se puede jugar las veces que se quiera con el fin de probar otras elecciones o para alcanzar puntuaciones más altas.

Finalmente, la plataforma tiene una sección para el profesor, en donde este puede monitorear el rendimiento de sus alumnos de manera global y de manera individual. De este modo puede darse cuenta de los puntajes obtenidos por los alumnos en cada episodio y en cada reto.
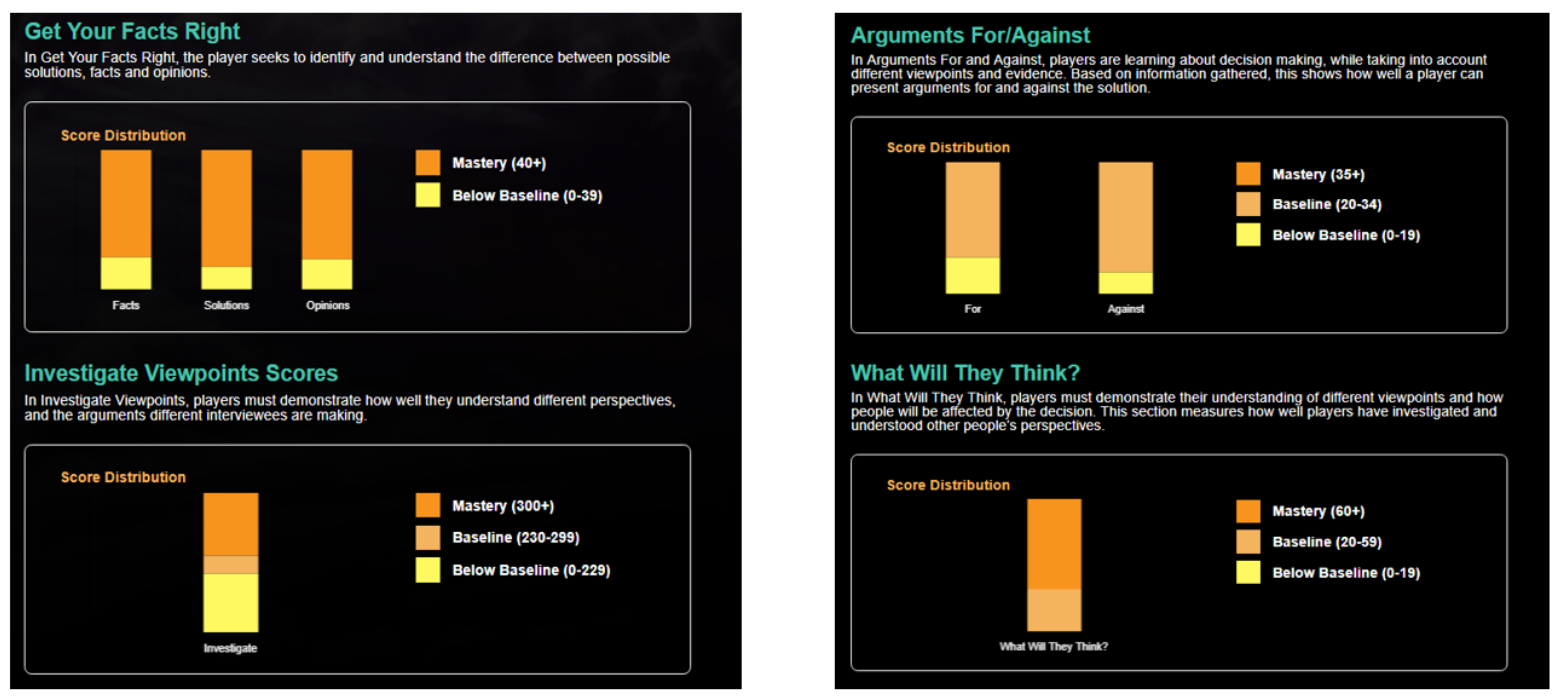

Figura 2. Seguimiento de habilidades argumentativas en Quandary Fuente: Learning Games Network, 2019 


\section{RESULTADOS}

Para comunicar los resultados obtenidos debe recordarse que los dos objetivos planteados fueron por una parte explorar el juego Quandary y su utilidad para la enseñanza de habilidades argumentativas básicas; y por otra parte observar la progresión de los estudiantes en el domino de habilidades argumentativas básicas (identificación de hechos, opiniones y soluciones y la clasificación de argumentos a favor y en contra) al interactuar con el entorno Quandary. También debe recordarse que metodológicamente se anunció un tratamiento mixto de los datos obtenidos, con una primera fase cualitativa y una segunda fase cuantitativa (Qual $\rightarrow$ Quan). En virtud de esto se dará cuenta del primer objetivo privilegiando el aspecto cualitativo y luego se abordará el segundo objetivo privilegiando el aspecto cuantitativo.

\subsection{Exploración del juego}

Respecto al primer objetivo, debe mencionarse que la exploración inicial de Quandary se llevó a cabo con ocho estudiantes de grado 10-3 a finales de agosto. Se hizo una demostración del entorno virtual del juego, encontrando una respuesta positiva ya que los alumnos mostraron interés en jugar.

Ante tal respuesta y antes de proponer el juego a todos los estudiantes del penúltimo grado, se detectaron las habilidades argumentativas que se potencian con Quandary y las herramientas de seguimiento que el entorno ofrece al profesor para hacer seguimiento a la progresión que va teniendo el alumno. Cabe a anotar que de las habilidades detectadas se eligieron dos consideradas básicas: por una parte el reconocimiento de hechos opiniones y soluciones y por otra la diferenciación de argumentos a favor $y$ argumentos en contra.

Es importante resaltar que en la exploración inicial se detectó que como en el juego se plantean "dilemas" o retos éticos en situaciones conflictivas por las que atraviesan los grupos de personas en ciertos momentos; se evidenció que aparte de enseñar a argumentar, el juego era compatible con la programación curricular para ese momento del año escolar, cuyo asunto era la reflexión ética en el área de filosofía y por tanto podía ayuda a reforzar los contenidos del plan de estudios.

Una semana después de la exploración inicial se prepararon unas diapositivas y se presentó el juego a los restantes alumnos del grado 10-3; se le entregó a cada uno su nombre de usuario y contraseña. También se hizo un ejercicio escrito de poner en situación, para hacer un diagnóstico de las habilidades a observar durante los episodios 
del juego. El ejercicio consistía en imaginar un conflicto en una comunidad, el cual los alumnos, en cuanto líderes, deberían ayudar a solucionar. De tal ejercicio lo que se pudo detectar es que no había total claridad para distinguir entre un hecho y una opinión; además que había más dificultad para plantear argumentos en contra que argumentos a favor. Durante la semana siguiente los alumnos entraron a Quandary y aunque el juego está diseñado para interactuar individualmente, algunos estudiantes se reunieron en una casa para jugar juntos. Viendo una nueva respuesta positiva, se decidió proponer el juego a los otros dos grupos del penúltimo grado de secundaria.

Se presentó el entorno Quandary al grupo 10-2 pero no se realizó la actividad diagnóstica y se decidió más bien explicar los cuatro retos del juego, dar las contraseñas y jugar una parte del primer episodio con ellos; de este modo también se percibió una positiva recepción de la propuesta. Sin embargo una alumna planteó la siguiente objeción “¿Cómo nos vamos a poner a jugar, si hay tantas cosas por hacer?” y curiosamente otras estudiantes secundaron la objeción presentada. Tal reacción puede dar a entender varias cosas que piensan algunos estudiantes acerca de la relación juego-estudio: que estudiar no es algo tan divertido como jugar; que la actividad propuesta no tiene que ver con el estudio; que primero es la obligación de estudiar y luego la diversión de jugar.

También se propuso el juego al grupo 10-1, pero como actividad diagnostica esta vez se planteó un caso que facilitara el análisis situacional por parte de los estudiantes. El caso era: Un grupo debe hacer un trabajo para el colegio pero el papá de una chica no la deja salir de la casa. Aunque la situación facilitó la implicación de los estudiantes en el análisis, los resultados son similares a los obtenidos con el grupo 10-3.

Después de 15 días exploración y presentación, los estudiantes iniciaron a jugar con espíritu competitivo pues veían que la plataforma hacia una clasificación general. Cabe anotar que de los 102 estudiantes de los tres grupos, 85 aceptaron participar; los otros 17 fueron nombrados monitores encargados de recolectar los puntajes obtenidos por los demás estudiantes y consignarlos en un formato para su posterior análisis. 


\begin{tabular}{|c|l|l|l|l|l|}
\hline Username & Ep 1 & \multicolumn{1}{|c|}{ Ep 2 } & Ep 3 & Ep 4 & Avg \\
\hline $10-16$ & 799 & 897 & 1025 & 3240 & 1118 \\
\hline $10-378$ & 375 & 630 & 630 & 2750 & 1096 \\
\hline $10-253$ & 580 & 1103 & 1050 & 2260 & 1042 \\
\hline $10-375$ & 560 & 780 & 920 & 2070 & 1022 \\
\hline $10-19$ & 675 & 920 & 930 & 1740 & 988 \\
\hline $10-369$ & 570 & 800 & 860 & 1650 & 970 \\
\hline $10-257$ & 560 & 450 & 680 & 1527 & 960 \\
\hline $10-373$ & 610 & 660 & 950 & 2290 & 927 \\
\hline $10-251$ & 500 & 360 & 780 & 1425 & 878 \\
\hline $10-120$ & 425 & 640 & 600 & 2220 & 862 \\
\hline
\end{tabular}

Ilustración 3. Consolidado de puntajes en Quandary Fuente: Learning Games Network, 20191

En la tercera semana de septiembre, es decir una semana después de haber propuesto la actividad a la totalidad de los estudiantes, 76 de los 85 estudiantes habían jugado el primer y segundo episodio de Quandary. En la semana siguiente la mayoría de estos estudiantes avanzaron hacia el segundo y tercer episodio.

Sin embargo al iniciar la primera semana de octubre se notaron problemas para acceder al juego y al tablero de control del profesor; tal error se debió a la sincronización de los usuarios con una actualización de la plataforma, según informaron las personas responsables de soporte técnico. A mitad de semana el impase se resolvió y se avisó a los estudiantes que podían volver a entrar sin problema al juego para terminar los episodios tres y cuatro, pero la respuesta no fue tan positiva. Adicionalmente la semana siguiente fue de receso estudiantil lo que generó una atmosfera poco favorable para reavivar el interés.

Al regresar del receso se dieron nuevamente indicaciones para que los estudiantes completaran los episodios; algunos lo hicieron, muchos no retomaron los que les faltaron y los que habían mostrado interés intentaban remontar los puntajes máximos que compañeros de diferentes salones habían alcanzado, lo cual generó una sana competencia; de hecho dos estudiantes lograron estar en el tablero de líderes de los jugadores de Quandary en el mundo.

\footnotetext{
${ }_{1}^{1}$ Al nombre de usuario que da la plataforma, se le superpuso manualmente el código asignado por el docente, en virtud de la protección de datos de los estudiantes.
} 


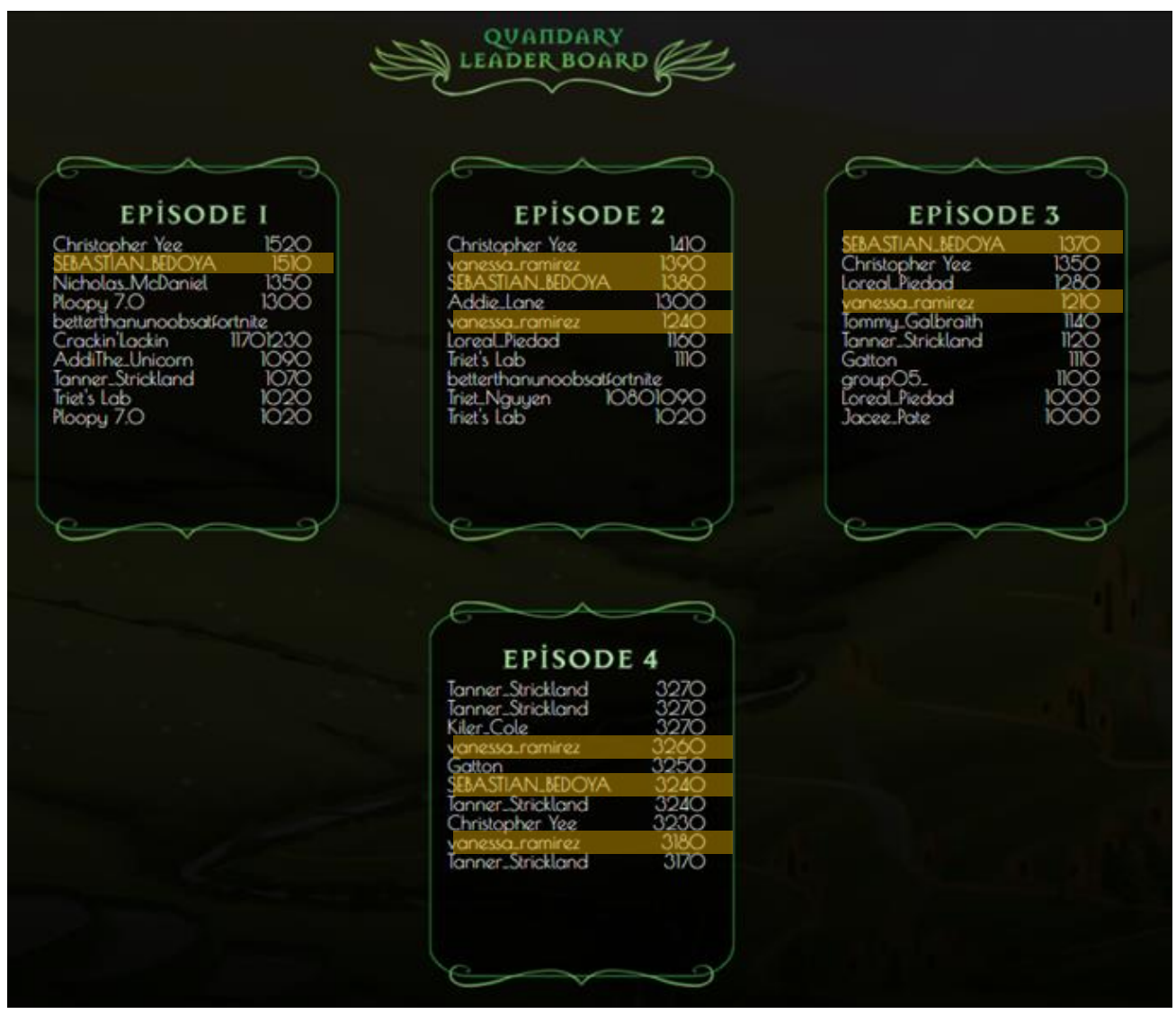

Figura 4. Tablero general de puntajes

Fuente: Learning Games Network, $2019^{2}$

Se nota pues que la falla técnica presentada hacia la mitad del proceso y el receso estudiantil influyeron para que la motivación decayera; tanto es así que en las tres semanas previas los estudiantes acumularon 152 horas de juegos, pero en las dos semanas posteriores se acumularon 38 horas adicionales. De hecho 40 de los 85 estudiantes que empezaron a jugar, no completaran los cuatro episodios.

\subsection{Progresión de los estudiantes}

Para analizar la progresión que los estudiantes mostraron en las habilidades de diferenciar hechos, opiniones y soluciones y clasificar argumentos a favor y argumentos en contra; se acudió al tablero de seguimiento de puntajes que la plataforma Quandary brinda al profesor, en donde se clasifican los resultados obtenidos de esta manera: 1) bajo la línea base, 2) entre la línea base y el nivel de domino, 3) sobre la línea de dominio. Tal clasificación hace posible establecer escalas para cada una de las

\footnotetext{
${ }^{2}$ Los nombres de los estudiantes no se quitaron ya que son datos de dominio público, y los alumnos no objetaron publicar sus nombres.
} 
habilidades observadas.

Es importante decir que la plataforma arrojó que 45 estudiantes culminaron los cuatro episodios, es decir $44 \%$ de todos los 103 estudiantes y $53 \%$ de los 85 que comenzaron a jugar. Sin embargo solo se tomaron datos de 44 estudiantes para el análisis estadístico dado que en un caso de los que terminaron, la interfaz de la plataforma no permitía visualizar ciertos puntajes obtenidos; algo análogo sucedió con otros participantes que no terminaron.

Entrando en materia hay que decir que se compararon los puntajes que estos 44 estudiantes obtuvieron en el primer episodio, con los obtenidos en el cuarto episodio respecto a las habilidades argumentativas básicas a observar. Adicionalmente se esperaba que, gracias a la práctica los puntajes obtenidos en el último episodio fueran mejores que los obtenidos en el primero.

\subsubsection{Identificación de hechos, opiniones y soluciones}

Para los puntajes obtenidos en esta habilidad y teniendo en cuenta que la escala va desde cero hasta cuarenta puntos, se establecieron 4 niveles:

- Bajo: 0 - 19 puntos

- Básico: 20 - 34 puntos

- $\quad$ Alto: 35 - 39 puntos

- Superior: 40 puntos

De acuerdo con los niveles establecidos y respecto a la identificación de hechos hay que decir que en 13 estudiantes, es decir el 29,55\% se observó el comportamiento esperado puesto que aumentaron su puntaje de un nivel básico o alto al nivel superior; excepto en un caso (10-133), en el que el estudiante aunque no cambió su nivel bajo, si lo subió un poco. 


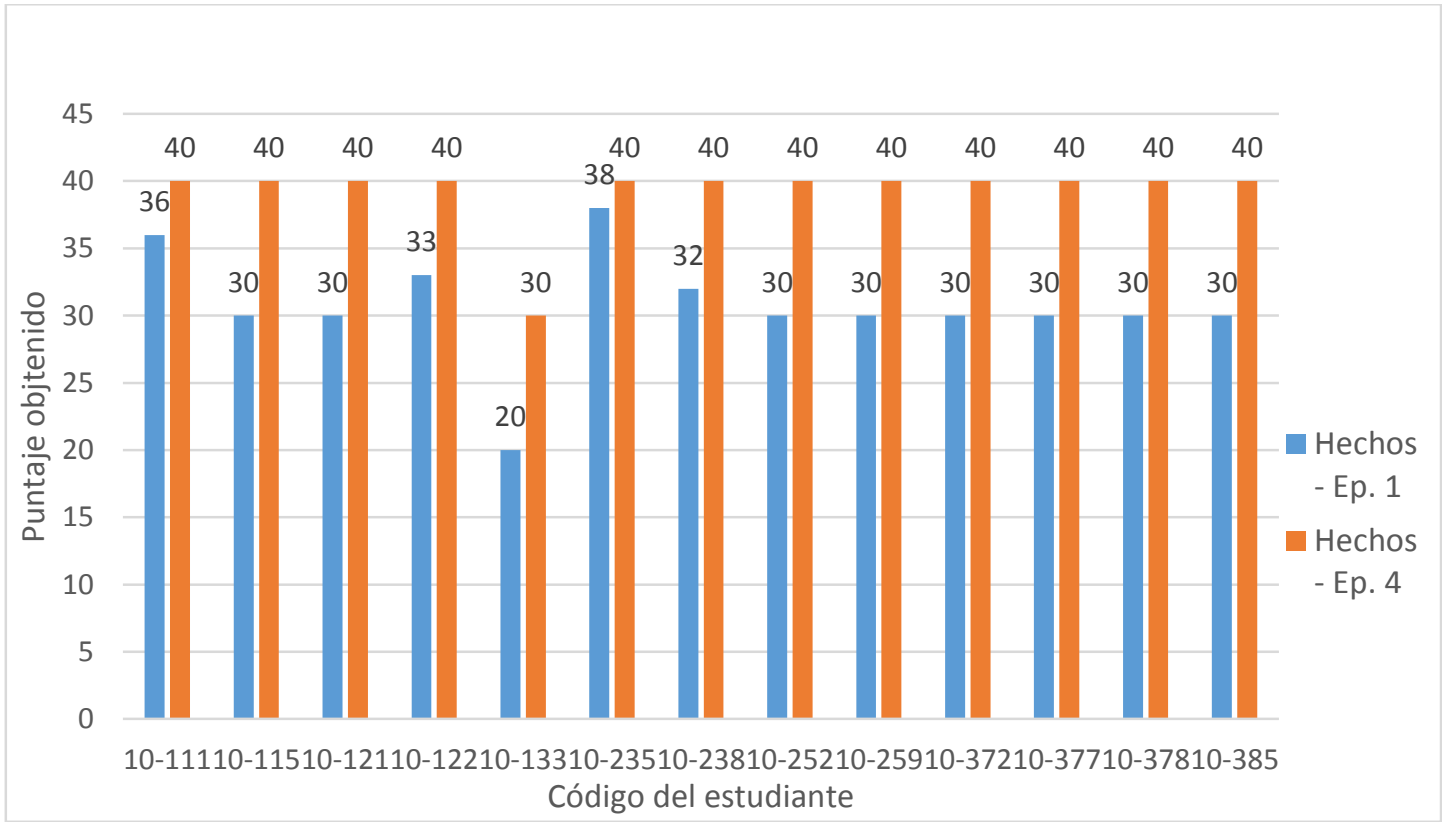

Ilustración 5. Comparativo identificación de hechos Fuente: elaboración propia

24 estudiantes tanto en el primer como en el cuarto episodio obtuvieron un puntaje de 40; por ende el 54,55\% se mantuvieron el nivel superior; 3 estudiantes se mantuvieron en su nivel alto; y 4 estudiantes no obtuvieron el comportamiento esperado, es decir el 9,09\%. En cuando a la identificación de opiniones, 14 estudiantes tuvo el comportamiento esperado, es decir el $31,82 \%$.

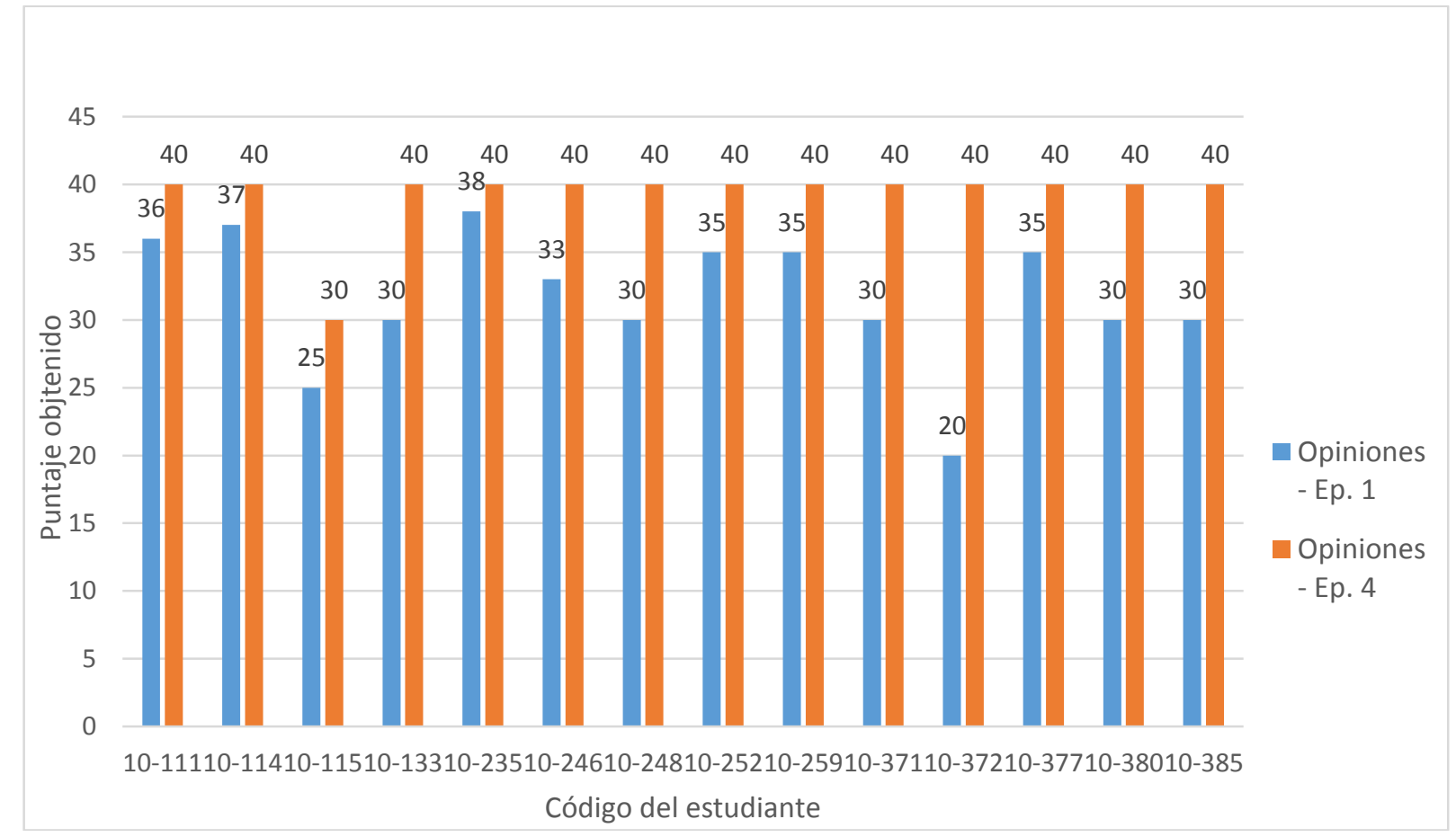

Figura 6. Comparativo identificación de opiniones Fuente: elaboración propia 
21 estudiantes (47,73\%) alcanzo nivel superior en los dos episodios comparados; y 9 $(20,45 \%)$ disminuyeron su nivel, es decir que no tuvieron el comportamiento esperado.

Respecto a la identificación de soluciones 13 estudiantes mejoraron su puntaje en el cuarto episodio. Es decir que el $29,55 \%$ tuvo el comportamiento esperado, tal como se aprecia en la siguiente imagen:

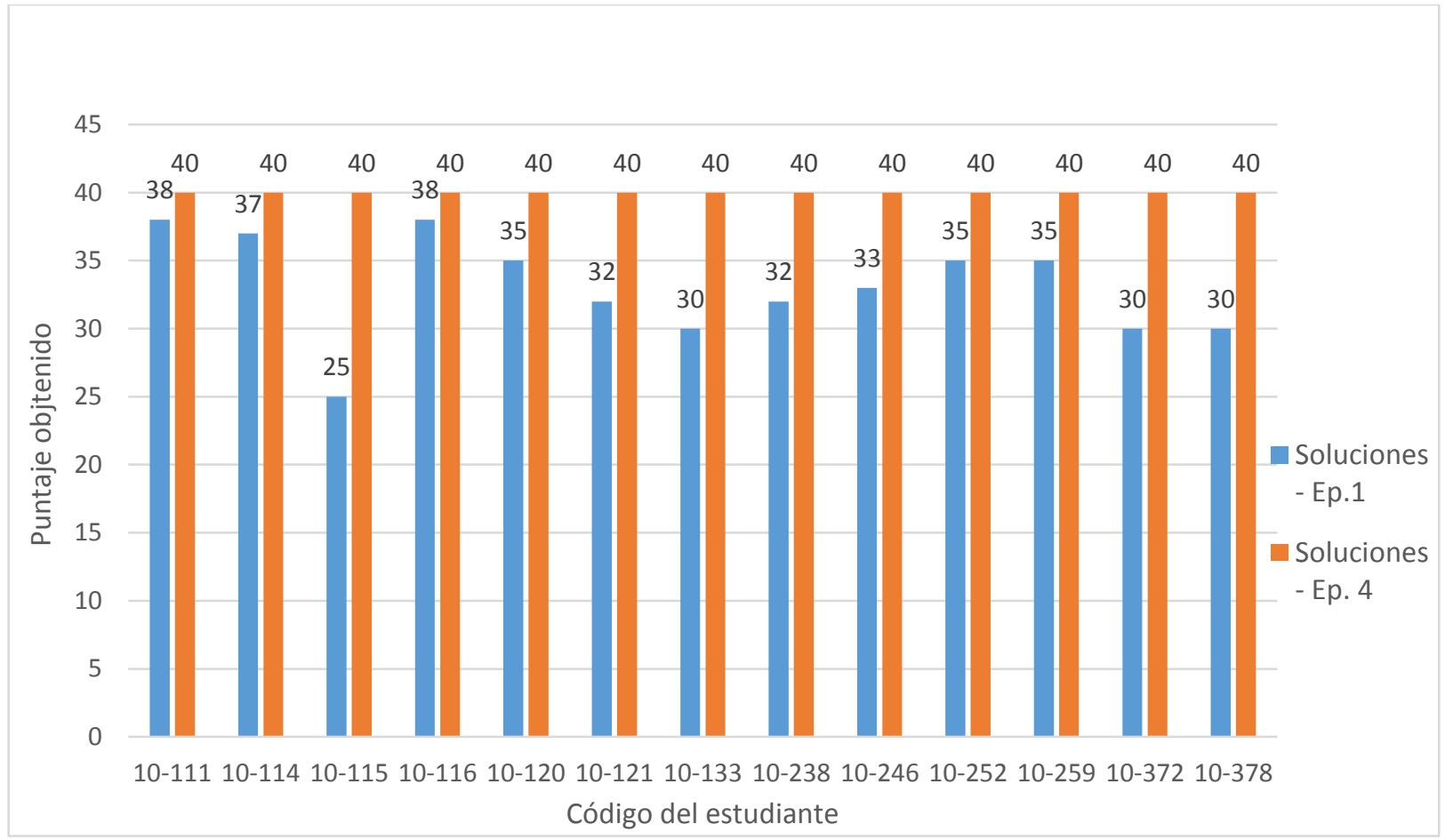

Figura 7. Comparativo identificación de soluciones Fuente: elaboración propia

Se observó también que hubo 27 estudiantes (61,36\%) que no variaron el nivel de superioridad (40 puntos); y otros $4(9,09 \%)$ que, como no subieron su puntaje, no tuvieron el comportamiento esperado.

\subsubsection{Clasificación de argumentos a favor y en contra}

Para la ubicación de los estudiantes en niveles respecto a esta habilidad inicialmente se pensó en la escala de 0 a 40 brindada por la plataforma Quandary; la cual suma los puntajes obtenidos al clasificar argumentos a favor y en contra. Sin embargo dado que se trata no de una habilidad sino de dos (clasificar argumentos a favor y clasificar argumentos en contra), se postuló una escala de 0 a 20 puntos para cada una de estas dos habilidades. Así las cosas se establecieron 4 niveles: 
- Bajo: 0 - 9 puntos

- Básico: 10 - 14 puntos

- $\quad$ Alto: $15-19$ puntos

- Superior: 20 puntos

De esta manera al observar la habilidad de clasificar argumentos a favor se observó que 14 estudiantes $(31,82 \%)$ tuvieron mejor puntaje en el cuarto episodio que en el primero

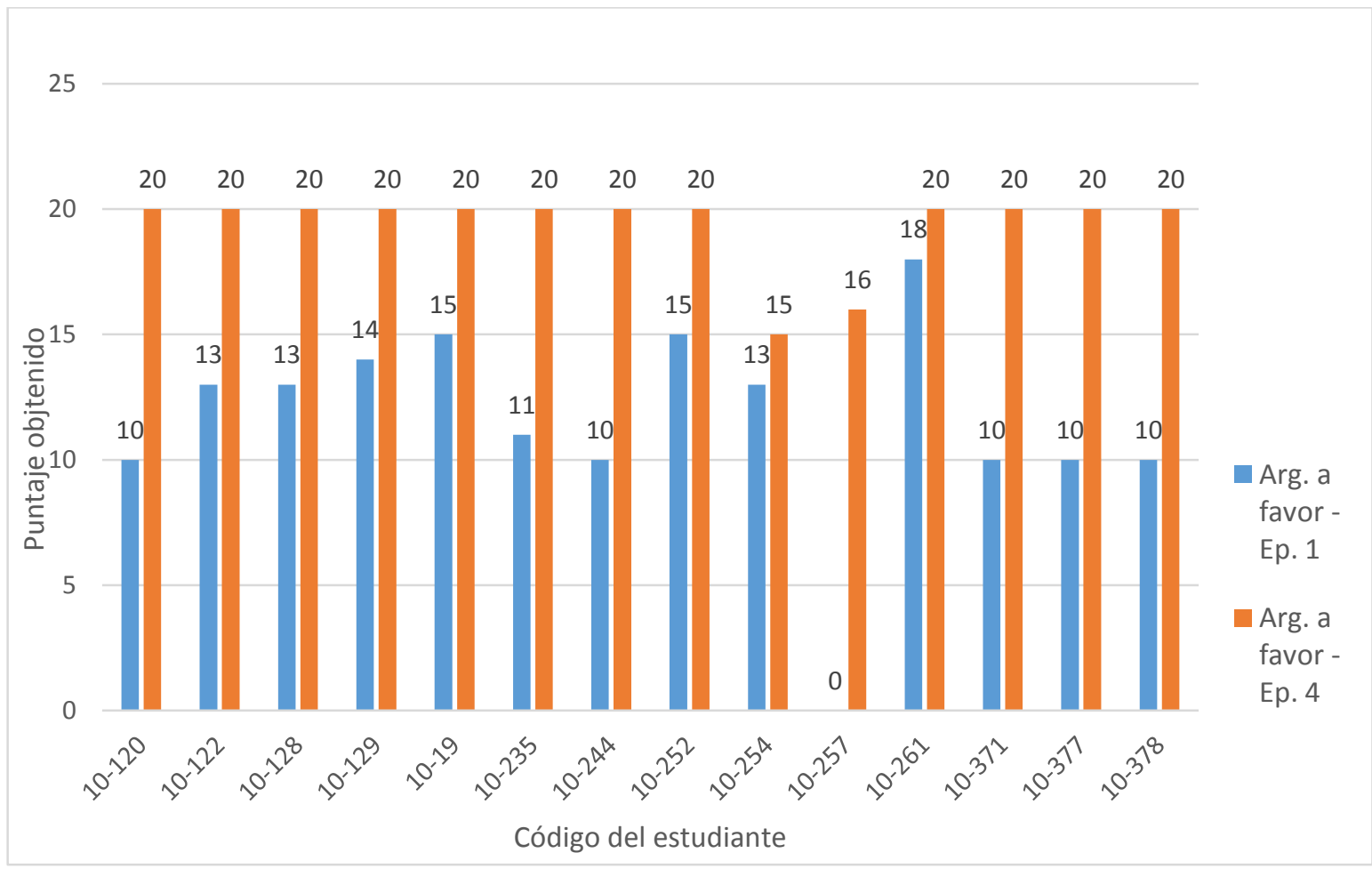

Figura 8. Comparativo clasificación argumentos a favor Fuente: elaboración propia

Cabe anotar que de igual manera otro $31,82 \%$ permaneció en su nivel superior y otro $31,82 \%$ no tuvo el comportamiento esperado porque en el último episodio obtuvo menos puntaje que en el primero. Adicionalmente 2 estudiantes (4,55\%) conservaron en su nivel básico.

Por lo que respecta a la última habilidad observada, que es clasificar argumentos en contra, se obtuvieron los siguientes resultados: 1 estudiante $(2,27 \%)$ conservó su nivel básico; 29 estudiantes (65,91\%) conservaron su nivel superior; 6 estudiantes $(13,64 \%)$ desmejoraron su puntaje en el episodio cuarto respecto al primero y 8 alumnos $(18,18 \%)$ tuvieron un comportamiento esperado dado que aumentaron su puntaje. 


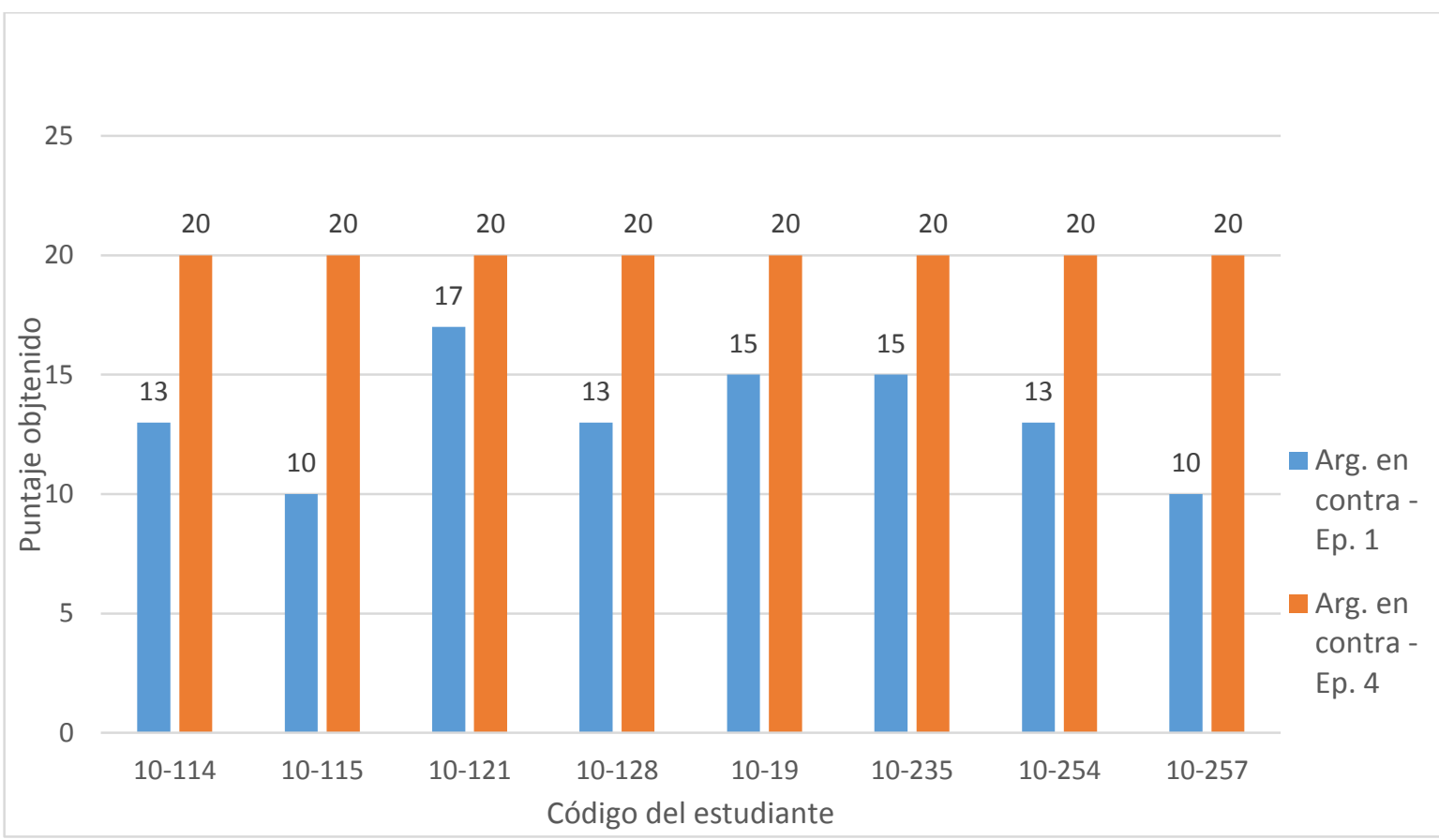

Figura 9. Comparativo clasificación de argumentos en contra Fuente: elaboración propia

Llama la atención constatar que la habilidad de clasificar argumentos en contra, parece estar menos desarrollada que la de clasificar argumentos a favor y aún menos que la de identificar hechos, opiniones o soluciones.

Para terminar esta presentación de resultados en la progresión de los estudiantes, resulta importante decir que 27 estudiantes $(61,36 \%)$ mejoraron su nivel de dominio en al menos una de las cinco habilidades argumentativas básicas observadas y 17 estudiantes (38,64\%) mantuvieron su nivel de máximo dominio en al menos una de las cinco habilidades observadas. Ningún estudiante presentó disminución en la totalidad de las habilidades observadas (identificación de hechos, identificación de opiniones, identificación de soluciones, clasificación de argumentos a favor y clasificación de elementos en contra).

\section{DISCUSIÓN}

\subsection{Enseñar a argumentar como línea investigativa}

Respecto a la línea de investigación adoptada hay que decir que a pesar de que hay una larga historia acerca del interés por los procesos de enseñanza-aprendizaje de habilidades argumentativas (Pérez-Echeverría, Postigo, \& Garcia-Mila, 2016) resulta importante, para la investigación y practica didáctica, especificar la diferencia entre los 
procesos de enseñanza y los procesos de aprendizaje de tales habilidades a partir de Hoyos Morales (2018).

Tal especificación pone en evidencia que son las personas las que aprenden o enseñan a argumentar y en tal sentido ayuda a poner el foco en el sujeto sobre el que recae la acción que o bien puede ser el alumno en el caso de observar el aprendizaje de habilidades argumentativas o bien puede ser el docente en el caso de observar la enseñanza de las habilidades en mención.

Este doble foco contribuye a profundizar trabajos como los de von Aufschnaiter, Erduran, Osborne, y Simon (2008), Schwarz (2009) y Schwarz \& Asterhan (2016) en los que se identifican como líneas investigativas sólo aprender a argumentar y aprender argumentando, dejando de lado el enseñar a argumentar y el enseñar argumentando. Precisamente el presente trabajo exploró la línea investigativa del enseñar a argumentar sin olvidar su relación con la línea del aprender a argumentar; relación que se sitúa a nivel de intencionalidad didáctica y no necesariamente de su realización.

Con la exploración emprendida se encontró que enseñar habilidades argumentativas es importante tanto para psicopedagogos como para estudiosos de la argumentación. Para los primeros esto se justifica porque resulta fundamental para alcanzar niveles cognitivos complejos (Bloom, 1956); para hacer visible el pensamiento (Perkins 1997) o desarrollar diversos tipos de inteligencias (Gardner, 2001); para desarrollar metacognición (Pintrich, 2002) o para pensar críticamente en la línea de Lipman o Paul \& Elder (2005). Por su parte para los estudiosos de la argumentación, las habilidades en cuestión desarrollan la racionalidad (Siegel, 1995); hacen posible analizar y evaluar discusiones críticas (van Eemeren \& Grootendorst, 2004); involucran los estudiantes en diálogos ricos y profundos (Kuhn, 2008); potencian el aprendizaje disciplinar (Muller Mirza y Perret-Clermont, 2009) o mejoran la práctica docente (Plantin, 2014).

Se ve pues que el enseñar a argumentar no se realiza en el vacío, ni se trata de un instrumentalismo o tecnicismo didáctico; se trata más bien de un enfoque con el que el docente se ve impelido a indagar acerca de su quehacer pedagógico y el alumno se abre a la posibilidad de una experiencia de aprendizaje que le haga crecer tanto en su dimensión cognitiva como afectiva (Hoyos-Morales, 2018, pág. 81).

Explorando la línea investigativa del enseñar a argumentar, también se encontró que para la investigación y la práctica didáctica es necesario definir cuales habilidades argumentativas enseñar y sus respectivos marcos conceptuales. En tal sentido 
aparecen diversas posibilidades, por ejemplo:

- Basándose en los procesos psicológicos superiores de la taxonomía de Bloom y su revisión hecha por Lorin \& Krathwohl (2001); se tendría como habilidades: precisión lógica, reconocer hechos, documentar pruebas e indicar falacias.

- Partiendo de la FpN, algunas habilidades argumentativas serían: formular preguntas e hipótesis, distinguir, comparar, generalizar, justificar propuestas, apreciar puntos de vista de otros o evaluar lógicamente.

- Acudiendo a Paul \& Elder, enseñar habilidades argumentativas implicaría enfatizar en los elementos que ellos identifican como propios del pensamiento crítico y los estándares intelectuales que cualifican tales elementos.

- Si se sigue el planteamiento de Kuhn, algunas habilidades a partir del patrón argumentativo de Toulmin serían plantear opiniones, generar razones, buscar evidencias, evaluar contra argumentaciones.

- Adoptar enfoques como el perelmaniano, el neodialéctico o el pragmadialéctico, ayudarían a plantear habilidades encaminadas al análisis y evaluación de las argumentaciones.

Para finalizar este primer apartado de la discusión, cabe anotar que metodológicamente se encontraron dos propuestas útiles para elegir cuales habilidades argumentativas enseñar. Por una parte la de Guzmán-Cedillo, et al (2013), en donde se plantea que la competencia argumentativa comprende actitudes, conocimientos y habilidades 0 destrezas automatizadas. Entre estas últimas están: enunciar una posición, organizar datos e ideas, evaluar la evidencia, encontrar contra argumentaciones y refutaciones. Por su parte Deane \& Song (2014) en su modelo de evaluación cognitiva de, por y para el aprendizaje, llamado CBAL (Cognitively Based Assessments of, for and as Learning) hablan de cuatro habilidades argumentativas: 1) comprender las solicitudes de la audiencia, 2) considerar puntos de vista, 3) evaluar argumentos y 4) presentar la argumentación. Estas habilidades son vistas no tanto como automatización de procesos sino como capacidades que permiten una acción experta en donde se involucra lo social, lo cognitivo y lo discursivo; lo que a su vez incluye los aspectos actitudinal, conceptual y procedimental que señalan Guzman-Cedillo et al. (2013). Así las cosas el segundo modelo aparece más incluyente que el de Cedillo.

\subsection{Progresión de habilidades argumentativas y juegos serios}

Decantarse por el modelo CBAL, a partir de un estudio de caso exploratorio como este, es razonable no solo porque tal modelo fue validado (Rijn, Graf, \& Deane, 2014). Si no 
también por su vinculación con los objetivos de este trabajo que eran explorar Quandary para la enseñanza de habilidades argumentativas básicas y observar la progresión de los estudiantes en el domino de habilidades argumentativas básicas al interactuar con dicho juego.

En primer lugar hay que resaltar que los trabajos de Deane, Song et al. (2014), están a la base del juego elegido (Quandary). No en vano las cuatro habilidades argumentativas del modelo CBAL mencionadas antes, se relacionan respectivamente con habilidades argumentativas trabajadas en Quandary: 1) diferenciar hechos de soluciones y de opiniones, 2) investigar puntos de vista, 3) diferenciar argumentos a favor o en contra, y 4) identificar acuerdos y desacuerdos respecto al argumento presentado. Cabe anotar estas habilidades coinciden con "las contrapartes empíricas de las cuatro etapas de discusión crítica: la situación inicial, los puntos de partida, los medios argumentativos y el resultado del discurso argumentativo" (van Eemeren F. , 2012, pág. 234)

En segundo lugar resulta razonable decantarse por el modelo CBAL por su vinculación con progresiones para el aprendizaje de la argumentación en las que se establecen objetivos intruccionales precisos y la manera en que los puede alcanzar el estudiante, a lo largo de todo el ciclo escolar (Deane, Song, Graf, \& van Rijn, 2013).

En virtud de lo anterior, cabe decir que acudir al modelo en mención ayuda a asumir el desafío de enseñar a argumentar que, según Kuhn et al (2016), es un gran reto para los maestros, quienes necesitan apoyarse mutuamente para su trabajo de planeación, ejecución y evaluación de actividades encaminadas a la enseñanza de la argumentación (Øgreid \& Hertzberg, 2009); sobre todo si se quiere que los jóvenes se desenvuelvan razonablemente en el ágora virtual (Ehrenworth, 2017).

Al hilo de lo anterior hay que decir que de acuerdo con los resultados obtenidos por los estudiantes en Quandary, las habilidades observadas aunque son básicas son disociables. Por ejemplo la habilidad para identificar hechos, opiniones y soluciones; se disocia en habilidad para identificar hechos, habilidad para identificar opiniones y habilidad para identificar soluciones. $Y$ la habilidad para clasificar argumentos a favor $y$ en contra se disocia en clasificar argumentos a favor y clasificar argumentos en contra. Tales disociaciones explican por qué algunos alumnos que mejoraron en la identificación de los hechos, no mejoraron en la identificación de opiniones; o alumnos que mejoraron en su habilidad para clasificar argumentos a favor no mejoraron en la clasificación de argumentos en contra. 
Ahora bien dado que, como se dijo antes, las habilidades trabajadas en Quandary coinciden con las contrapartes empíricas de las cuatro etapas del modelo pragmadialéctico de discusión crítica; puede decirse que las primeras tres habilidades disociadas están implicadas en la segunda etapa de la discusión critica (identificación de los puntos de partida), etapa que es más descriptiva que argumentativa. Las otras habilidades disociadas corresponden con la tercera etapa (surgimiento de medios argumentativo), que es la propiamente argumentativa.

Así las cosas, según las habilidades en las que se destacaron los alumnos estos podrían perfilarse de diferentes formas: 1) los que tienden a ser más hábiles para identificar hechos, opiniones o soluciones; estos serían más descriptivos que argumentativos. 2) los que tienden a clasificar mejor los argumentos a favor y en contra; estos serían más argumentativos que descriptivos. 3) los que muestran habilidad tanto para identificar (hechos, opiniones y soluciones) como para clasificar (pros y contras); estos serían descriptivos-argumentativos. 4) los que tienen dificultad para ambos tipos de habilidades; los cuales aún no serían ni descriptivos ni argumentativos.

Según lo anterior, el seguimiento a progresiones de habilidades argumentativas a través de juegos serios como Quandary tiene una función diagnóstica y una formativa. Diagnostica porque ayuda al profesor a darse cuenta del perfil y el nivel en el que están los estudiantes y formativa porque ayuda a planear actividades al interior de estrategias formativas.

También se puede decir que a partir de los resultados obtenidos, el uso de Quandary fue positivo no solo porque los estudiantes o mejoraron o mantuvieron su nivel máximo al comparar los puntajes obtenidos en el primer y último episodio; sino porque, en el sondeo de opinión realizado, varios de ellos (12 de 31) manifestaron que el reto de identificar hechos, opiniones y soluciones «aclaró lo que hacemos todos los días con nuestras acciones y las decisiones que tomamos y cómo las realizamos buscando opiniones y hechos que nos ayuden a tomar una decisión mejor» (Estudiante 10-246).

Otros estudiantes manifestaron que «el juego nos ayuda a saber pensar $y$ razonar mejor para saber solucionar los problemas, ya que en eso consiste la vida, también el tomar buenas decisiones» (Estudiante 10-256).

Llama la atención que varios estudiantes describieron este juego serio como entretenido y curioso bien porque «tenía situaciones conflictivas» (Estudiante 10-13 y 10-133), bien porque «enganchaba a la historia» (Estudiante 10-16). Cabe anotar que 
algunos lo encontraron aburrido bien porque «los hacia leer varias veces» (Estudiante 10-116), bien porque su «categoría de juegos es muy diferente» (Estudiante 10-120).

Finalmente cabe anotar que cuando se les preguntó acerca de las estrategias usadas, la mayoría respondió que se esforzaban por leer cuidadosamente; sin embargo otros fueron más específicos y declararon que tomaron apuntes (Estudiante 10-113, 10$115,10-249,10-255)$ o que jugaron varias veces intentando diferentes opciones o que se reunieron con compañeros para discutir que opciones eran mejores (Estudiante 10259).

\subsection{Límites y perspectivas de este trabajo}

Los límites de este trabajo se refieren no tanto a los problemas técnicos con la plataforma, ni a los tiempos planeados para que los estudiantes interactuaran con Quandary. Se refieren más bien a asuntos metodológicos en los que se relacionan los objetivos y los elementos estructurantes de este estudio de caso: juego serio, nuevas tecnologías y habilidades argumentativas.

En este sentido resultó útil acudir no solo al modelo CBAL, sino al contruccionismo de Papert, en especial a tres de las ideas que están detrás de su laboratorio de Aprendizaje construccionista: 1) diversión difícil, 2) estamos en el mundo digital y 3) el aprender a aprender o metacognición.

Tales ideas dan un marco unitario a los tres elementos articuladores de este trabajo, mencionados antes. En primer lugar el juego serio se relaciona con la diversión difícil en términos de problemas a solucionar o retos a afrontar; de hecho para Papert «el aprendizaje es esencialmente difícil; sucede mejor cuando uno está profundamente involucrado en actividades difíciles y desafiantes» (Papert, 1998, pág. 88). En segundo lugar la presencia de nuevas tecnologías en educación se relaciona con la importancia que Papert da al mundo digital no desde el punto de vista tecnocéntrico sino desde el convencimiento que las TIC son herramientas del intelecto y por ello «están ligadas a la revalorización de procesos de enseñanza-aprendizaje y a una revolución en lo cognitivo» (Baptista Lucio, 2008, pág. 15). En tercer lugar la importancia de enseñar habilidades argumentativas básicas, por ejemplo para desarrollar metacognición, se relaciona con el construccionismo de Papert el cual centrándose en el aprender a aprender, permite a los estudiantes «conocer sus habilidades e identificar las herramientas cognitivas pertinentes para su propio proceso educativo, y según sus niveles de desarrollo» (Aparicio Gómez \& Ostos Ortiz, 2018, pág. 118). 
Sin embargo, lo anterior no explicita las relaciones entre los elementos articuladores de este caso; lo cual representa un límite de este trabajo. En tal sentido para futuras investigaciones modelos como el TPACK (Koehler, Mishra, \& Cain, 2016) serían quizás más útiles dado que plantea la importancia de tener en cuenta: 1) conocimiento del contenido (Contend Knowledge) que se refiere a lo que se enseña, y se relaciona aquí con las habilidades argumentativas básicas. 2) El conocimiento pedagógico (Pedagogical Knowledge) que se refiere al cómo se enseña, a los marcos metodológicos o técnicas didácticas; lo cual tiene que ver, en este trabajo, con el modelo CBAL de progresiones en el aprendizaje de habilidades argumentativas básicas. 3) Conocimiento sobre tecnología (Technological Knowledge) que se refiere a la pregunta con que instrumento o soportes se enseña; y se relaciona en este estudio de caso con el uso inteligente de los juegos o plataformas on-line.

Pero además el modelo TPACK incluye las relaciones entre los tres tipos de conocimientos mencionados: conocimiento pedagógico del contenido, conocimiento tecnológico del contenido, conocimiento tecnológico pedagógico y conocimiento tecnológico pedagógico del contenido.

Conocimiento pedagógico del contenido (PCK): tiene que ver con el asunto didáctico. Por ejemplo en este trabajo se refiere a la manera en que los juegos serios ayudan a dar cuenta de las progresiones en el aprendizaje de habilidades argumentativas básicas en determinado momento del ciclo escolar.

Conocimiento tecnológico del contenido (TCK): se refiere a «la forma en que los contenidos... pueden verse afectados por la aplicación de la tecnología» (GarcíaValcárcel \& Martín del Pozo, 2016, pág. 70) en este caso la manera en que Quandary potencia el aprendizaje de ciertas habilidades argumentativas o limita el de otras.

Conocimiento tecnológico pedagógico (TPK): hace referencia a «las estrategias pedagógicas generales que se pueden realizar a través de las tecnologías» (GarcíaValcárcel \& Martín del Pozo, 2016, pág. 71); tiene que ver para el presente caso con la forma en que Quandary, gracias a sus características, resulta ser una herramienta útil y eficaz.

Conocimiento tecnológico pedagógico del contenido: se «refiere a la comprensión que surge de la interacción entre los saberes de contenido, pedagogía y tecnología» (Koehler, Mishra, \& Cain, 2016, pág. 17). En tal sentido habría que reflexionar, a partir del modelo TPACK acerca de las relaciones entre modelo CBAL, juegos serios y las líneas investigativas que surgen de la relaciones entre enseñanza, 
aprendizaje y argumentación.

Para terminar es interesante señalar que la versión 2019 de Quandary ofrece materiales para que los profesores trabajen no solo habilidades argumentativas, sino también contenidos disciplinares y aprendizaje basado en proyectos; lo cual abre posibilidades para profundizar en las cuatro líneas investigativas que relacionan argumentación y educación; es decir enseñar a argumentar, aprender a argumentar, enseñar argumentando y aprender argumentando.

\section{REFERENCIAS}

Aberdein, A. (2010). Virtue in Argument. Argumentation, 24, 165-179. doi:10.1007/s10503-0099160-0

Aparicio Gómez, O. Y., \& Ostos Ortiz, O. L. (2018). El constructivismo y el construccionismo. Revista interamericana de investigación, educación y pedagogía , 11(2), 115-120. doi:10.15332/25005421

Baptista Lucio, P. (2008). Seymur Papert: parábolas para explicar el debate sobre las tic en la educación. Revista panamericana de pedagogía, 14, 11-22. Obtenido de http://portalderevistasdelaup.mx/revistapedagogia/index.php/pedagogia/issue/view/19

Baquero, R. (1997). Vigotsky y el aprendizaje escolar (Segunda ed.). Buenos Aires: Aique Grupo Editor S. A.

Bloom, B. (1956). Taxonomy of Educational Objectives. The Classification of Educational Goals. LONGMANS.: Michigan.

Brown, A. C. (2016). Classroom Community and Discourse: How Argumentation Emerges during a Socratic Circle. Dialogic Pedagogy, 4. doi:https://doi.org/10.5195/dpj.2016.160

Ciftci, S. (February de 2018). Trends of Serious Games Research from 2007 to 2017: A Bibliometric Analysis. Journal of Education and Training Studies, 6(2). Obtenido de http://jets.redfame.com

Creswell, J. W. (2009). Research Desing. Qualitative, Quantitative, and Mixed Methods Approaches. Los Angeles: Sage.

Deane, P., \& Song, Y. (2014). A case study in principled assessment design: Designing assessments to measure and support the development of argumentative reading and writing skills. Psicología Educativa, 20(2), 99-108. doi:10.1016/j.pse.2014.10.001

Deane, P., Song, Y., Graf, E., \& van Rijn, P. (Noviembre de 2013). Using Argumentation Learning Progressions to Support Teaching and Assessments of English Language Arts. Research \& Development Connections, 1-14. Obtenido de https://www.ets.org/research/policy_research_reports/publications/periodical/2013/jrgw

Ehrenworth, M. (2017). Why Argue? Educational Leadership, 74(5), 35-40. Obtenido de https://eric.ed.gov/?id=EJ1128258

Fuentes Bravo, C., \& Santibañez Yañez, C. (2010). Diseñando debates: preliminares para un enfoque dialógico y crítico. En A. Cattani, \& U. Cundari (Ed.), Argomentare le proprie ragioni. Organizzare, condurre e valutare un dibattito (pág. 200). Padova: Loffredo editore. University press.

García-Valcárcel, A., \& Martín del Pozo, M. (Abril de 2016). ¿Se sienten preparados los graduados en maestro primaria para afrontar la profesión docente? Bordón. Revista de pedagogía, 69-84.

Gardner, H. (2001). Estructuras de la Mente. La Teoría de Las Inteligencias Múltiples. Bogotá: Fondo de Cultura Económica.

Gardner, H., \& Davis, K. (2013). The App Generation. New Haven: Yale University Press. 
Gimeno Sacristán, J., \& Pérez Gómez, A. I. (1992). Comprender y transforma la enseñanza (Undécima ed.). Madrid: Morata.

Glassner, A. (2017). Evaluating arguments in instruction: Theoretical and practical directions. Thinking Skills and Creativity,, 95-103. Obtenido de 10.1016/j.tsc.2017.02.013

Guzmán-Cedillo, Y. I., Flores-Macías, R. d., \& Tirado-Segura, F. (2013). Desarrollo de la competencia argumentativa en foros de discusión en línea: una propuesta constructivista. Anales de Psicología, 29(3), 907-916. doi:https://dx.doi.org/10.6018/analesps.29.3.175681

Hoyos-Morales, J. I. (10 de Diciembre de 2018). Implicaciones de la argumentación en clase para la enseñanza. Estudio de caso en un bachillerato en ciencias sociales. Tesis doctoral. Mencion Cum Laude, 326. Segovia, Valladolid, España: Universidad de Valladolid. Obtenido de http://uvadoc.uva.es/handle/10324/33459

Jackson, B., \& Wallin, J. (2009 de 2009). Rediscovering the "Back-and-Forthness" of Rhetoric in the Age of YouTube. College Composition and Communication, 61(2), 374-396. Obtenido de http://cccc.ncte.org/cccc/ccc/issues/v61-2

Koehler, M. J., Mishra, P., \& Cain, W. (2016). ¿Que son los saberes tecnológicos y pedagógicos del contenido (TPACK)? Virtualidad, educación y ciencia, 6(10), 9-23. Obtenido de https://revistas.unc.edu.ar/index.php/vesc/article/view/11552

Kuhn, D. (2008). Education For Thinking . Cambridge, Massachusetts: Harvard University Press.

Kuhn, D., Hemberger, L., \& Khait, V. (2016). Dialogic argumentation as a bridge to argumentative thinking and writing. Infancia y Aprendizaje, 39(1), 24-48. Obtenido de http://www.tandfonline.com/doi/full/10.1080/02103702.2015.1111608

Learning Games Network. (2019). Curriculum Intro. Obtenido de www.quandarygame.org/sites/default/files/QuandaryCurriculumlntro.pdf

Leitão, S. (2012). O trabalho com argumentação em ambientes de ensino-aprendizagem: um desafio persistent. Uni-pluri/versidad, 12(3), 23-37.

Lipman , M., Sharp, A. M., \& Oscanyan, F. S. (1992). La filosofía en el aula. Madrid: Ediciones de la Torre.

Lorin , A. W., \& Krathwohl , D. (Edits.). (2001). A Taxonomy for Learning, Teaching and Assessing: a Revision of Bloom's Taxonomy of Educational Objectives. New York: Longman.

Muller Mirza, N., \& Perret-Clermont, A.-N. (Edits.). (2009). Argumentation and Education: Theoretical Foundations and Practices. Dordrecht: Springer. doi:10.1007/978-0-38798125-3_1

Øgreid, A., \& Hertzberg, F. (2009). Argumentation in and Across Disciplines: Two Norwegian Cases. Argumentation, 451-468.

Ormrod, J. E. (2005). Aprendizaje humano (4 ed.). (A. J. Escudero, \& M. Olmos Soria, Trads.) Madrid: PEARSON EDUCACIÓN, S.A.

Papert, S. (Junio de 1998). Does Easy Do It? Children, Games, and Learning. Game Developer magazine, 87-88. Obtenido de https://www.gdcvault.com/gdmag

Papert, S. (2013). Eight Big Ideas Behind the Constructionist Learning Lab. En G. Stager, \& S. Libow Martinez, Invent To Learn: Making, Tinkering, and Engineering in the Classroom. Obtenido de http://stager.org/articles/8bigideas.pdf

Paul, R., \& Elder, L. (2005). Estándares de Competencia para el Pensamiento Crítico. Obtenido de Foundation for Critical Thinking: www.criticalthinking.org

Pérez-Echeverría, M. P., Postigo, Y., \& Garcia-Mila, M. (2016). Argumentación y educación: apuntes para un debate. Infancia y Aprendizaje / Journal for the Study of Education and Development, 39(1), 1-24. doi:http://dx.doi.org/10.1080/02103702.2015.1111607

Perkins, D. (1997). ¿Como hacer visible el pensamiento? (P. León, \& M. X. Barrera, Edits.) Escuela de Graduados en Educación de la Universidad de Harvard. Obtenido de http://conexiones.dgire.unam.mx/wpcontent/uploads/2017/11/perkins_david_como_hacer_visible_el_pensamiento.pdf

Perkins, D. (2001). La escuela inteligente. Del adiestramiento de la memoria a la escuela inteligente. (G. Ventureira, Trad.) Barcelona: Gedisa, S.A. 
Pintrich, P. R. (2002). The Role of Metacognitive Knowledge in Learning, Teaching, and Assessing. THEORY INTO PRACTICE, 41(4).

Plantin, C. (Julio de 2014). Lengua, argumentación y aprendizajes escolares. Tecné Episteme $Y$ Didaxis, 36, 95-114. doi:10.17227/01213814.36ted95.114

Rijn, P., Graf, A., \& Deane, P. (2014). Empirical recovery of argumentation learning progressions in scenario-based assessments of English language arts. Psicología Educativa, 20(2), 109-115. doi:https://doi.org/10.1016/j.pse.2014.11.004

Rodriguez Gomez, G., \& Ibarra Sáiz, M. S. (12-14 de Noviembre de 2014). Desarrollo de la competencia evaluadora en estudiantes universitarios a través de juegos de simulación. Obtenido de https://www.oei.es/historico/congreso2014/memoriactei/1240.pdf

Rugelj, J. (octubre-diciembre de 2014). Los videojuegos serios en la educación en Informática. Novática(230). Obtenido de:

https://www.researchgate.net/profile/Joe_Rugelj/publication/271516658_Los_videojueg os_serios_en_la_educacion_en_Informatica/links/54ca79b60cf2517b755e0d73.pdf

Schwarz, B. (2009). Argumentation and learning. En N. Muller Mirza, \& A. N. Perret-Clermont, Argumentation and education. Theoretical foundations and practices (págs. 91-126). Dordrecht Heidelberg London New York: Springer Science+Business Media. doi:10.1007/978-0-387-98125-3

Schwarz, B. B., \& Asterhan, C. S. (2016). Argumentation for Learning: Well-Trodden Paths and Unexplored Territories. Educational Psychologist, 51, 164-187: doi:10.1080/00461520.2016.1155458

Siegel, H. (1995). Why Should Educators Care about Argumentation? Informal Logic, 17(2), 159176.

van Der Heide, J., Juzwik, M., \& Dunn, M. (2016). Teaching and Learning Argumentation in English: A Dialogic ApproachTheory into Practice. 55(4), 287-293: doi:https://doi.org/10.1080/00405841.2016.1208069

van Eemeren, F. (2012). Maniobras estratégicas en el discurso argumentativo (1 ed.). (C. Santibañez Yañez, \& M. Molina, Trads.) Madrid - Mexico: Plaza y Valdéz.

van Eemeren, F., \& Grootendorst, R. (2004). A Systematic Theory of Argumentation: The pragmadialectical approach. Amsterdam: Cambridge University Press.

von Aufschnaiter, C., Erduran, S., Osborne, J., \& Simon, S. (2008). Arguing to learn and learning to argue: Case studies of how students' argumentation relates to their scientific knowledge. Journal of Research in Science Teaching, 45, 101-131: doi:10.1002/tea.20213

Warley, J. (Noviembre de 2011). El hacer argumentativo. Pedagogía y teoría de la argumentación: Entrevista a Christian Plantin. Anclajes, XV(2), 103-112.

Yin, R. K. (2003). Case Study Research. Desing and Methods. London: Sage Publications Inc.

AGRADECIMIENTOS: Se agradece, en primer lugar, a todas las directivas del colegio Antonio Holguín Garcés (Cartago - Colombia) por facilitar las condiciones básicas para la realización del trabajo empírico; en segundo lugar, a los estudiantes del penúltimo curso de educación media (año 2018) por su esfuerzo y dedicación. Por otra parte, se agradece al proyecto EuricaEuropean Commission 2013-2587/001-001-EM Action 2, en cuyo marco se realizó la investigación "Implicaciones de la argumentación en clase para la enseñanza"; pues dicho trabajo proveyó el marco teórico del presente artículo. Finalmente, se agradece tanto a los profesores Bartolomé Rubia, Luis Torrego y Suyapa Martínez, de la Universidad de Valladolid; como a los docentes José Rubiel Bedoya y Luz Adiela Valencia, del colegio Holguín Garcés, por sus sugerencias y comentarios.

JORGE IVAN HOYOS: Doctor en Filosofía por la Universidad de Antioquia (Colombia) y Doctor en Investigación transdisciplinar en educación por la Universidad de Valladolid (España). Sus intereses investigativos se centran en la relación de la argumentación con la enseñanza y el aprendizaje. 